\title{
Cooperation and cognitive radio
}

\author{
O. Simeone, J. Gambini(*), Y. Bar-Ness \\ CWCSPR, NJIT \\ University Heights, NJ 07102, USA \\ Email: osvaldo.simeone, yeheskel.barness@njit.edu
}

\author{
U. Spagnolini \\ ${ }^{(*)}$ DEI, Politecnico di Milano \\ P.za L. da Vinci, 32, I-20133 Milan, Italy \\ Email: spagnoli@elet.polimi.it
}

\begin{abstract}
Cooperation is increasingly regarded as a key technology for tackling the challenges of a practical implementation of cognitive radio. In this paper, we first give a brief overview of the envisioned applications of cooperative technology to cognitive radio, distinguishing among cooperative sensing for detection of the primary activity, cooperative transmission between secondary nodes and cooperative transmission of primary traffic by secondary users (cognitive relaying). Then, we focus on the latter scenario and investigate a simple wireless network, where one secondary transmitter has the option to relay traffic of the primary. Assuming that the primary is oblivious to the presence of the secondary (thus excluding the possibility of spectrum leasing), the secondary transmitter optimizes transmission/ relaying parameters towards the goal of maximizing the rate towards the secondary receiver. Numerical results are provided in order to discuss the advantage and limits of cognitive relaying.
\end{abstract}

\section{INTRODUCTION}

Cognitive radio is a new paradigm in wireless communications that promises an enhanced utilization of the limited spectral resource. The basic idea is to employ a hierarchical model, where primary (licensed) and secondary (unlicensed) users coexist in the same frequency spectrum. Primary users have privileged access to the common channel and can dispose of it according to traffic and quality-of-service needs, limited only by the available radio interfaces and regulations. In a typically envisioned scenario, primary users are expected to be oblivious to the presence of secondary terminals, thus behaving as if they had exclusive access to the spectrum. Therefore, secondary terminals aim at exploiting the idle periods of primary (usually referred to as spectral holes) in an opportunistic fashion ${ }^{1}$. Alternatively, primary users can actively grant channel access to the secondary users for a fraction of the temporal/ spectral resource in exchange for some form of retribution (spectrum leasing). In this paper, as in most of the current literature on cognitive radio, we focus on the first scenario (see [1] [2] for an overview on cognitive radio).

The main challenges towards the implementation of the principle of cognitive radio appear to be:

- Primary activity detection at the secondary nodes (spectrum sensing): secondary users need to monitor the avail-

\footnotetext{
${ }^{1}$ Pre-cancellation schemes inspired by information theory [3] could also be employed by the secondary users. Accordingly, transmission by the secondary would occur at the same time and on the same bandwidth of the primary activity without causing any interference. However, these schemes are based on strict assumptions regarding the availability of instantaneous channel state information at the secondary nodes (see [4] [5]).
}

able spectrum (or a portion of it, if there are energy consumption constraints) in order to be able to detect spectral holes. A typical way to address the problem is to look for primary transmissions by using a signal detector. The trade-off between probability of false alarm and missed detection then amounts in this scenario to trading missed transmission opportunities for an increased interference level on the primary [2] [6]. The main issue is to enable quick and effective detection at all the secondary nodes that can potentially interfere with the primary transmission. Notice that given the receivercentric definition of interference, a major problem is also that of detecting or locating primary receivers [7]. The impact of practical limitations in spectrum sensing on the performance of cognitive radio from a system perspective have been investigated in [8];

- Transmission opportunity exploitation: once a spectral hole has been identified (within the error probability set by the receiving operating curve of the detector), secondary users need to exploit the transmission opportunity so as to satisfy two conflicting objectives: (i) making their activity transparent to the primary users (according to a defined criterion for transparency, such as keeping the interference level below a given threshold); (ii) maximize their own performance in terms of the desired quality of service indicator (e.g., rate, delay, etc.). Secondary users might be competing for the resource, or cooperating in order to improve efficiency and fairness of resource sharing. In both cases, analysis can be usefully carried out using concepts from various field such as information theory [9] and game theory [10].

The solution of both problems illustrated above can greatly benefit from cooperation among different terminals. In the following, we first provide a brief overview on the applications of the collaborative technology ${ }^{2}$ to cognitive radio (Sec. II), and then focus on a specific example that shows the advantages of cooperation in the form of relaying of primary traffic via a secondary node (Sec. III). This examples builds on the recent paper [11], where the basic idea of cooperative relaying from a secondary node has been introduced.

\footnotetext{
${ }^{2}$ In this paper, we will use the terms "cooperation" and "collaboration" interchangeably.
} 

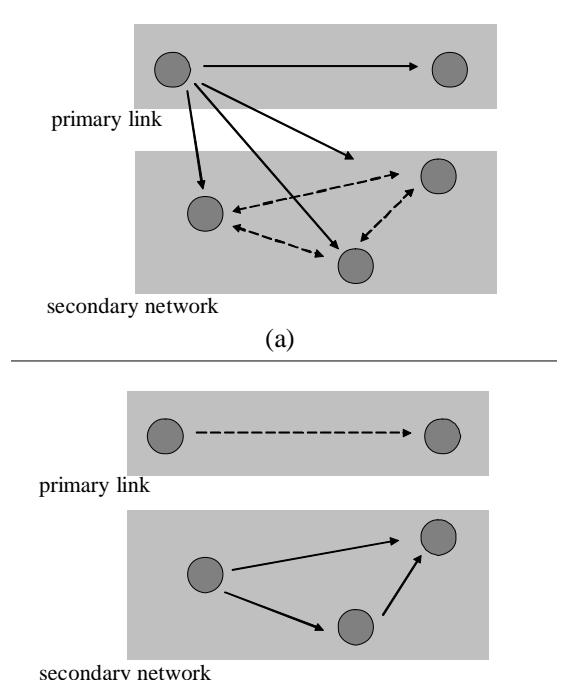

(b)

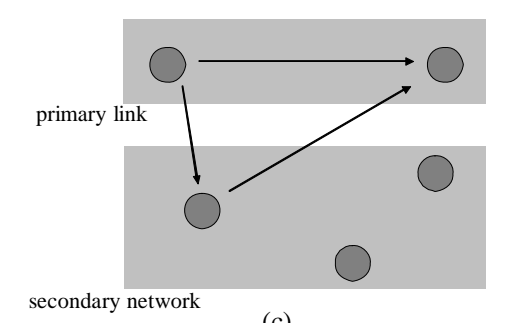

(c)

Fig. 1. A simple cognitive wireless network with one primary link and a secondary network of three nodes: (a) Cooperative sensing; (b) Cooperative transmission between secondary terminals; (c) Cognitive relay.

\section{CoOperation IN COGNITIVE RADiO}

The performance of both primary activity detection and transmission opportunity exploitation (see previous section) has been recently shown to improve drastically if cooperation is deployed. In the following, we consider separately the two problems and provide a brief description of different available solutions.

\section{A. Cooperative sensing in cognitive radio}

In order to enhance the performance of primary (transmitter) detectors, a natural solution that is able to cope with different shadowing/ fading conditions is cooperation. The basic idea is to employ distributed detection at the secondary nodes: each node measures (e.g., through an energy detector) the local received signal, then local signals are exchanged (possibly to a central decision point) and finally a global decision on the primary activity is achieved (fig. 1-(a)). This approach is clearly robust to possible unbalance of the channel qualities of different secondary users and shows to achieve a drastic improvement of the receiving operating curve [12].

An alternative approach to cooperative sensing is interestingly based on cooperative transmission among the secondary users and is discussed in the next section (see fig. 1-(b)).

\section{B. Cooperative transmission in cognitive radio}

Cooperative transmission in its basic forms refers to the information theoretic model of the relay channel, where one node (the relay) forwards the transmission another node (the source) towards the intended destination. Performance advantages achievable from collaboration arise from: (i) power gains, that can be harnessed if the relay happens to be in a convenient location, typically halfway between source and destination; (ii) diversity gains, that leverage the double path followed by the signal (direct source-destination and relay transmissions) [13]. In the context of cognitive radio, cooperative transmission can give rise to two different basic scenarios, as explained in the following.

1) Cooperative transmission between secondary users: In this scenario, a secondary user acts as relay from the transmission of another (source) secondary terminal (see fig. 1-(b)). General considerations valid for cooperative transmission can be applied in this case (see, e.g., [13]) with the important caveat that secondary nodes need to continuously monitor the channel for possible transmissions by the primary. Interestingly, [12] proposed to use cooperative transmission as a means to enhance the sensing process as well. The main idea is to let the secondary relay node amplify and forward the received signal since the latter contains not only the transmission from the secondary source, but also, if present, the signal from the primary. This forwarding then allows the secondary destination to improve the local detection of the primary user in a scenario where the relay is placed approximately halfway between primary and secondary destination.

2) Cognitive relay: Apart from cooperation between secondary users, a different form of cooperative transmission can be envisioned where a secondary users has the possibility to relay the traffic of a primary transmitter towards the intended destination (see fig. 1-(c)). The rationale of this choice is that helping the primary to increase its throughput entails (for a fixed demand of rate by the primary) a diminished transmission time of the primary, which in turns leads to more transmission opportunities for the secondary [11] (see also [4] [5] where cognitive relaying is shown to increase the capacity region). Therefore, while cooperation between secondary users aims at increasing the secondary throughput for a given spectral hole, cognitive relaying pursues an enhanced throughput by increasing the probability of transmission opportunities. In the next section, we discuss a simple scenario with one primary and one secondary link where the transmitter may act as relay (also referred to as cognitive interference channel, see fig. 2) in order to show the advantages of cognitive relaying.

\section{COGNITIVE RELAY}

As introduced in the previous section, cognitive relaying appears to a be a promising approach for improving the throughput of secondary nodes by increasing the transmission opportunities. In this section, we further investigate this concept, by considering the scenario in fig. 2, first studied in [11] under a simplified protocol. 


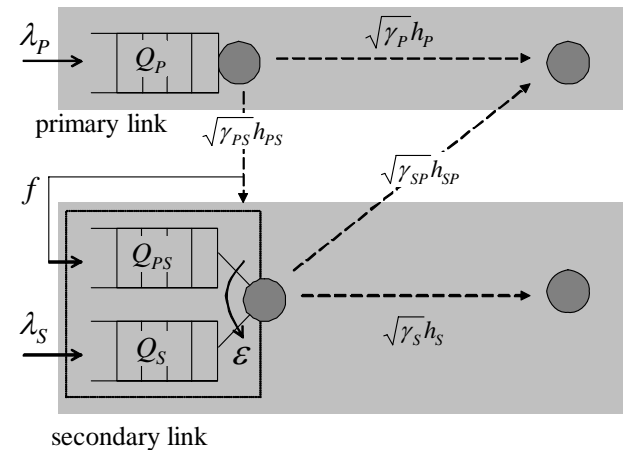

Fig. 2. A simple cognitive relay model with one primary and one secondary links.

\section{A. System model}

Referring to fig. 2, both primary and secondary transmitting nodes are equipped with an infinite queue in which incoming packets are stored. All packets have the same number of bits, and their transmission time coincides with a time slot, which we consider as our reference time unit. The arrivals of packets at each transmitting station are independent and stationary processes, with $\lambda_{P}$ (packets/ slot) being the mean arrival rate at the primary queue and $\lambda_{S}$ (packets/ slot) the mean arrival rate at the secondary queue. The primary transmitter accesses the channel whenever it has a packet in its queue $Q_{P}(t)$ at the beginning of the slot $t$, being oblivious to the presence of a secondary link. On the contrary, the secondary transmitter sends a packet to its destination in a given slot only if it senses an idle channel according to the spectrum sensing scheme described in Sec. III-C (and if it has a packet to transmit in its queues and $Q_{P S}(t)$, see below). Notice that the analysis in [11] did not explicitly model spectrum sensing.

Whenever a primary packet is not correctly received by the intended destination but is instead decoded at the secondary transmitter, the latter has the choice to store the packet in a separate queue $Q_{P S}(t)$ for later forwarding to the secondary transmitter (cognitive relaying). In this paper, we assume that, under the conditions mentioned above, the secondary transmitter accepts the primary packet with a probability $f$ (see Sec. III-B for further discussion on this packet acceptance control mechanism that was not considered in [11]). Moreover, whenever an idle slot is detected, the secondary transmits a packet from the queue $Q_{S}(t)$ containing its own packets with scheduling probability $\varepsilon$ and from the queue $Q_{P S}(t)$ with complementary probability $1-\varepsilon$.

As for the signalling protocols, we consider that each receiving node sends the respective transmitting node an ACK message in case of a correct reception or a NACK message in case of an erroneous reception. A packet reception error requires retransmission. Notice that, in this analysis, the overhead introduced in the system by the transmission of ACK-NACK messages is considered negligible and therefore not modelled ${ }^{3}$.

Independent Rayleigh block-fading channels are assumed between every pair of nodes. In particular, the complex channel gains on the $i$ th link (where subscript $i$ identifies transmitterreceiver pairs as illustrated in fig. 2) at the $t$ th slot read $\sqrt{\gamma_{i}} h_{i}(t)$ where $h_{i}(t)$ is a zero-mean unit-variance stationary process and $\gamma_{i}$ is the average (time-invariant) channel power gain. Moreover, the primary transmitter employs, without loss of generality, unit power, while the secondary transmits with power $P_{S} \leq 1$. Packet transmission is considered successful if the instantaneous signal-to-noise ratio is above given thresholds $\beta_{P}$ for the primary link and $\beta_{S}$ for the secondary. Notice that in case of missed detection of primary activity, the secondary interferes with the primary transmission, and in this case primary transmission is successful if the signal-to-noiseplus-interference ratio is above the threshold $\beta_{P}$.

1) Problem definition: The primary transmitter in fig. 2 selects its arrival rate $\lambda_{P}$ within its own stability region, being oblivious to the presence of the secondary. On the contrary, the secondary transmitter adapts its transmission mode to best accomplish two conflicting goals: (i) making its activity transparent to the primary link and (ii) maximizing its own stable throughput $\mu_{S}$ (defined as the number of packets per slot such that the secondary queue is stable for any $\lambda_{S} \leq \mu_{S}$ ). We follow the approach presented in [11], where the constraint on transparency requires the stability of the queue of the primary transmitter. In other words, we say that the activity of the secondary node is transparent to the primary if the queue of the latter remains stable. The transmission mode of the secondary transmitter is here defined by the transmit power $P_{S}$, the scheduling probability $\varepsilon$, the packet acceptance ratio $f$ (see also next section) and the threshold $\alpha$ of the spectrum sensing detector (see Sec. III-C).

In order to decide its transmission mode, the secondary transmitter is assumed to know the average (or long-term) channel parameters $\left(\gamma_{P}, \gamma_{S}, \gamma_{P S}, \gamma_{S P}\right)$, under the premise that, before starting transmission, the secondary node has estimated these parameters during an observation period of a few time slots. Notice that $\gamma_{P}, \gamma_{S}$ and $\gamma_{S P}$ can be estimated from energy measurements on the respective channels (considering feedback from the receivers, e.g., for ACK/ NACK), whereas the knowledge of $\gamma_{P}$ is more critical and can be reasonably calculated from geometrical consideration according to a path loss model.

Remark 1: Cognitive relaying aims at enhancing the secondary throughput via the increase of transmission opportunities for the secondary. It should be noted that this is achieved by increasing the overall energy consumed by the secondary (i.e., power times the number of transmitted packets), since the latter has to deliver not only its traffic but also some packets from the primary. In this paper, we only consider power constraints and thus do not tackle this issue. However, in

\footnotetext{
${ }^{3}$ Notice that, for a primary packet accepted by the secondary, the primary might receive two acknowledgments for the same packet. In this case, it will simply consider the packet as correctly received if at least one acknowlegment is positive.
} 
energy-limited scenario, the performance of cognitive relaying requires further investigation.

\section{B. Packet acceptance control}

Cognitive relaying prescribes the possibility for the secondary transmitter to forward primary packets that have not been successfully received at the intended destination but have instead been decoded at the primary transmitter. This event is clearly likely to happen if the direct average channel power $\gamma_{P}$ is sufficiently smaller that the power gain $\gamma_{P S}$ between primary and secondary transmitters. However, in order to be able to actually deliver the primary packets to the destination, thus avoiding overflow of the queue $Q_{P S}(t)$, the channel gain $\gamma_{S P}$ needs to be sufficiently large, as pointed out in [11]. The solution studied here prescribes a packet acceptance control mechanism where the secondary, based on the knowledge of the average channels (see previous section), only accepts a fraction of packets $f$ out of the primary packets that have not been successfully received at the intended destination but have instead been decoded at the primary transmitter. It is expected that an optimal $f$ will be small whenever channel gain $\gamma_{S P}$ is not sufficiently large in order to avoid congestion of queue $Q_{P S}(t)$. Further discussion on this point is provided in Sec. IV.

\section{Spectrum sensing}

According to the basic principle of cognitive radio, at the beginning of each slot, the secondary transmitter senses the channel in order to find whether it is employed by the primary or not. In this paper, we explicitly model the detection process by using an energy detector [15] [16]. More precisely, at the beginning of each slot, the secondary node measures $m$ samples (at symbol rate) of the received signal in order to detect the activity of the primary. The secondary transmitter compares the output of the energy detector with a particular threshold $\alpha$ in order to decide whether the primary node is transmitting or not. The secondary transmitter is interested in choosing the optimum detection threshold $\alpha$ as the result of a trade-off between limiting the interference on the primary (probability of missed detection) and increasing secondary channel access (probability of false alarm).

\section{Optimizing the transmission mode}

Optimizing the transmission mode $\left(P_{S, \varepsilon}, f, \alpha\right)$ of the secondary as explained in Sec. III-A.1 requires to write an optimization problem that can be obtained by extending the analysis in [11]. Here, for lack of space we omit the analysis but we remark that the extending of the results in [11] to an optimization over the further degrees of freedom of packet acceptance ratio $f$ and detection threshold $\alpha$ is relatively straightforward. We concentrate instead on some numerical results that illustrate the performance of cognitive relaying.

\section{NumericAl RESUlts}

In this section, we present some numerical results in order to get insight into the performance of cognitive relaying and,

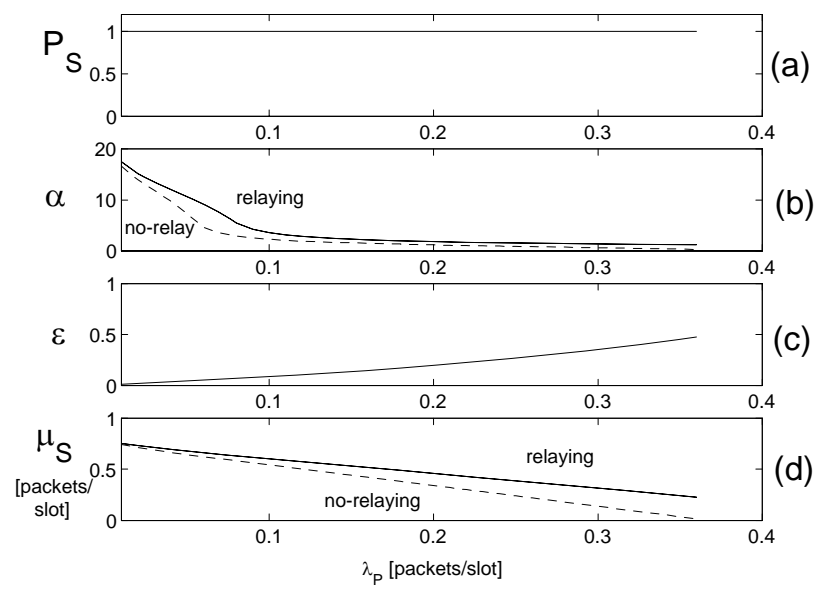

Fig. 3. Optimal choice for power $P_{S}$, detection threshold $\alpha$ and scheduling probability $\varepsilon$, and corresponding maximum stable throughput $\mu_{S}$ versus the arrival rate $\lambda_{P}$ selected by the primary node $\left(\beta_{P}=\beta_{S}=4 \mathrm{~dB}, \gamma_{P}=4 \mathrm{~dB}\right.$, $\gamma_{S}=\gamma_{P S}=\gamma_{P S}=10 \mathrm{~dB}, m=5$ ).

in particular, into the conditions under which this solution provides performance advantages. We set the number of training symbols for the energy detector to $m=5$, the outage thresholds to $\beta_{P}=\beta_{S}=4 \mathrm{~dB}$, and $\gamma_{S}=10 \mathrm{~dB}$.

Fig. 3 compares the performance of the simple cognitive scenario in fig. 2 for two cases where the secondary can (solid lines) and cannot (dashed lines) act as a relay for the primary traffic. In particular, we compare the two scenarios in terms of optimum choices for secondary transmit power $P_{S}$, detection threshold $\alpha$ and scheduling probability $\varepsilon$ (only applicable to relaying) and in terms of maximum stable throughput of the secondary node $\mu_{S}$. Here we set $f=1$ (no packet admission control) for simplicity, and other parameters are as follows: $\gamma_{P}=4 \mathrm{~dB}, \gamma_{P S}=\gamma_{P S}=10 \mathrm{~dB}$. The secondary maximum stable throughput $\mu_{S}$ clearly decreases with the primary arrival rate $\lambda_{P}$ according to the hierarchical model of cognitive radio In particular, the arrival rate $\lambda_{P} \simeq 0.36$ coincides with the maximum primary stable throughput $\mu_{P}$ and, at this point, $\mu_{S}=0$ if no relaying is deployed (see [11] for details). Moreover, in this example, the primaryto-secondary and secondary-to-primary channel power gains $\left(\gamma_{P S}\right.$ and $\gamma_{P S}$, respectively) are sufficiently larger than the direct primary channel $\gamma_{P}$, so that cognitive relaying leads to relevant secondary throughput gains, especially for large primary rate $\lambda_{P}$ (notice, for instance, that a non-zero $\mu_{S}$ is achieved even for $\lambda_{P}>0.36$ ). Finally, it can be seen that with cognitive relaying the optimal detection threshold $\alpha$ is larger than in the case of no relaying: this implies that relaying allows the secondary to choose a less conservative point on the receiving operating curve of its detector and thus to attempt access to the channel more often (notice also that the optimal power $P_{S}$ equals the maximum value 1 in both cases).

We are now interested in evaluating the limits of cognitive relaying. Consider a scenario where the direct primary channel is $\gamma_{P}=7 \mathrm{~dB}$ and the primary-to-secondary and secondary 


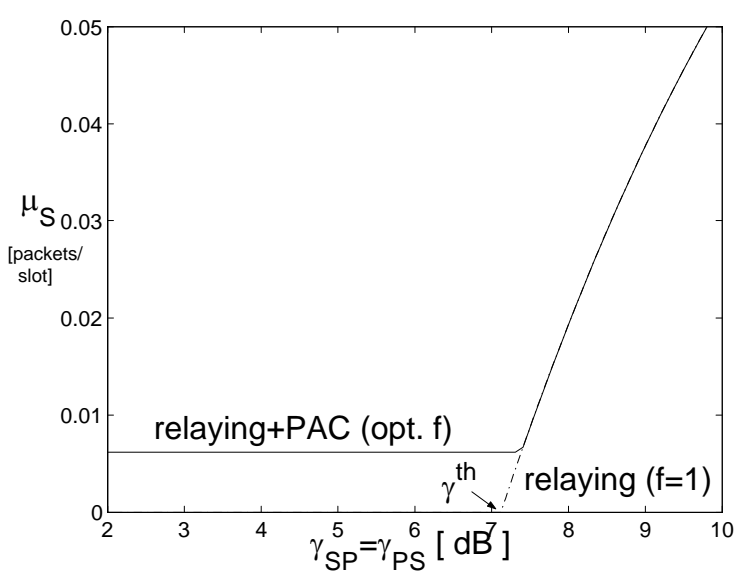

Fig. 4. Maximum throughput of the secondary user $\mu_{S}$ for a fixed $\lambda_{P}=$ $\mu_{P}^{\max }$ versus the value of the channel parameter $\gamma_{S P}$ for $f=1$ (relaying) and optimized $f$ (packet acceptance control, PAC) $\left(\beta_{P}=4 \mathrm{~dB}, \beta_{S}=4 \mathrm{~dB}\right.$, $\left.\gamma_{P}=7 \mathrm{~dB}, \gamma_{S}=10 \mathrm{~dB}, m=5, \lambda_{P}=0.36\right)$

to primary channels $\gamma_{P S}$ and $\gamma_{S P}$ take different values. Fig. 4 shows the maximum throughput of the secondary $\mu_{S}$ for cognitive relaying and $\lambda_{P}=0.36$ (see discussion above) considering both the case $f=1$ (no packet access control) and optimized packet acceptance ratio $f$. It is seen that for for channel gains $\gamma_{S P}$ and $\gamma_{P S}$ large enough $\left(>\gamma^{t h} \simeq \gamma_{P}=\right.$ $7 \mathrm{~dB}$ ), the secondary is able to relay traffic from the primary efficiently, thus creating transmission opportunities for its own traffic and increasing its own throughput. However, if $\gamma_{S P}$ and $\gamma_{S P}$ are not larger than this threshold $\left(<\gamma^{t h} \simeq \gamma_{P}\right)$ and there is no packet acceptance control $(f=1)$, the secondary is not able to deliver all the extra-traffic coming from the primary and the throughput of the secondary is zero. However, optimizing the packet acceptance ratio $f$ allows to obtain a non-zero secondary throughput. The conclusions above are further corroborated by fig. 5 where we consider again $\gamma_{P S}=$ $\gamma_{S P}$ and evaluate the minimum $\gamma_{S P}$ and $\gamma_{P S}$ (i.e., $\gamma^{t h}$ ) that allows a non-zero throughput for relaying without packet acceptance control $(f=1)$, for different values of $\gamma_{P}$. In particular, $\gamma^{\text {th }} / \gamma_{P}$ is shown to increase with $\gamma_{P}$, suggesting that advantages of cognitive relaying are more difficult to harness for larger $\gamma_{P}$. Moreover, the lower figure shows that the corresponding secondary throughput of packet acceptance control for this threshold condition is decreasing with $\gamma_{P}$.

\section{CONCLUSIONS}

In this paper, we have discussed some aspects of the interplay of cooperation and cognitive radio technologies. In particular, we have focused on an interesting instance of cooperative transmission, where a secondary node acts as a relay for the primary traffic. It has been shown that, under certain conditions on the network topology, cognitive relaying is effective in enhancing secondary throughput by increasing the number of transmission opportunities.
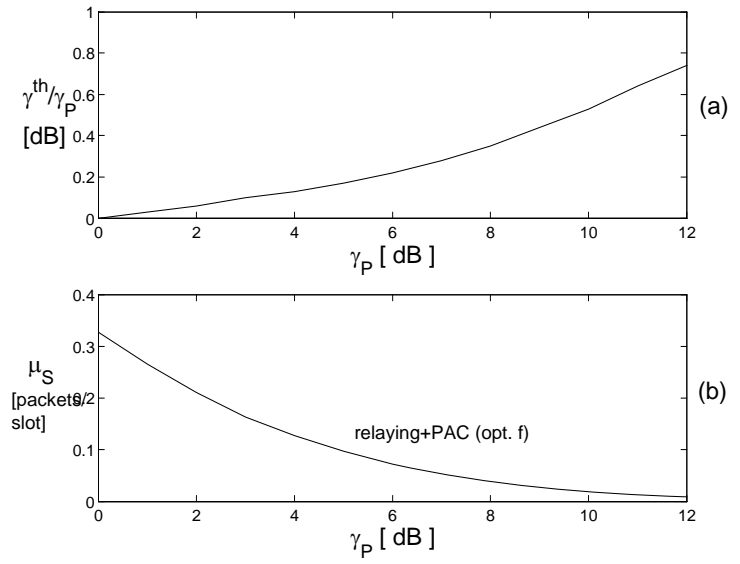

Fig. 5. (a) Minimum value of $\gamma_{S P}=\gamma_{P S}$ that allows a non-zero throughput for cognitive relaying with $f=1$ (i.e., $\gamma^{\text {th }}$ ), versus $\gamma_{P}$. (b) Secondary throughput $\mu_{S}$ with packet acceptance control for $\gamma_{S P}=\gamma_{P S}=\gamma^{\text {th }}$ versus $\gamma_{P}\left(\beta_{P}=4 \mathrm{~dB}, \beta_{S}=4 \mathrm{~dB}, \gamma_{S}=10 \mathrm{~dB}, \lambda_{P}=0.36, m=5\right)$.

\section{REFERENCES}

[1] S. Haykin, "Cognitive radio: brain-empowered wireless communications," IEEE Journal on Selected Areas Commun., vol. 23, no. 2, pp. 201-220, Feb. 2005.

[2] Q. Zhao and B.M. Sadler, "Dynamic Spectrum Access: Signal Processing, Networking, and Regulatory Policy," submitted to IEEE Signal Processing Magazine, September, 2006.

[3] M. Costa, "Writing on dirty paper," IEEE Trans. Inform. Theory, vol. 29, pp. 439-441, May 1983.

[4] N. Devroye, P. Mitran and V. Tarokh, "Limits on communications in a cognitive radio channel," IEEE Commun. Magazine, vol. 44, no. 6, pp. 44-49, June 2006.

[5] A. Jovicic and P. Viswanath, "Cognitive radio: an information-theoretic perspective," submitted [available on-line: http://lanl.arxiv.org/PS_cache/cs/pdf/0604/0604107.pdf]

[6] Y. Chen, Q. Zhao and A. Swami, ”Joint design and separation principle for opportunistic spectrum access," in Proc. Asilomar Conf. on Signals, Systems and Computers, 2006.

[7] B. Wild and K. Ramchandran, "Detecting primary receivers for cognitive radio applications," in Proc. of IEEE DySPAN, Nov. 2005.

[8] A. Sahai, N. Hoven and R. Tandra, "Some fundamental limits on cognitive radio," in Proc. Allerton Conference on Communication, Control, and Computing, October 2004.

[9] R. Etkin, A. Parekh, and D. Tse, "Spectrum Sharing for Unlicensed Bands," in Proc. IEEE DySPAN, Nov. 2005.

[10] J. Neel, J. Reed, R. Gilles, "The Role of Game Theory in the Analysis of Software Radio Networks," in Proc. SDR Forum Technical Conference, 2002.

[11] O. Simeone, U. Spagnolini and Y. Bar-Ness, "Stable throughput of cognitive radios with and without relaying capabilities," to appear on IEEE Trans. Commun [http://www.elet.polimi.it/upload/simeone/subcognitive.pdf].

[12] A. Ghasemi and E. S. Sousa, "Collaborative Spectrum Sensing for Opportunistic Access in Fading Environments," in Proc. IEEE DySPAN, Nov. 2005.

[13] A. Nosratinia, T. E. Hunter and A. Hedayat, "Cooperative communication in wireless network," IEEE Commun. Magazine, vol. 42, no. 10, pp. 68-73, Oct. 2004.

[14] G. Ganesan and Y. Li, "Cooperative Spectrum Sensing in Cognitive Radio Networks," in Proc. IEEE DySPAN, Nov. 2005.

[15] H. Urkowitz, "Energy detection of unknown deterministic signals," in Proc. of the IEEE, vol. 55, no. 4, pp.523-531, April 1967.

[16] F. F. Digham, M.-S. Alouini and M. K. Simon, "On the energy detection of unknown signals over fading channels," in Proc. IEEE ICC, pp. 35753579, May 2003. 